\title{
A rare case of patient with neurofibromatosis type 1 in a genotype-phenotype correlation revealing a submicroscopic deletion on the long arm of chromosome 17
}

Vityala Yethindra ${ }^{1}$, Tugolbai Tagaev ${ }^{2}$, Elmira Mainazarova ${ }^{3}$, Cholpon Dzhumakova ${ }^{4}$, and Asel Namazbekova ${ }^{4}$

${ }^{1}$ International Higher School of Medicine, International University of Kyrgyzstan

${ }^{2}$ I K Akhunbaev Kyrgyz State Medical Academy

${ }^{3}$ Kyrgyz-Russian Slavic University

${ }^{4}$ National Center of Oncology and Hematology

February 8, 2021

\begin{abstract}
We are reporting a case of neurofibromatosis type 1 in a genotype-phenotype correlation and chromosomal microarray test revealed a submicroscopic deletion on the long arm of chromosome 17, which is associated with a more severe phenotype. The presence of a more severe phenotype warrants precise monitoring of complications.
\end{abstract}

Title of the article: A rare case of patient with neurofibromatosis type 1 in a genotype-phenotype correlation revealing a submicroscopic deletion on the long arm of chromosome 17

\section{AUTHORS}

Vityala Yethindra $^{1^{*}}$, Tugolbai Tagaev ${ }^{2}$, Elmira Mainazarova $^{3}$, Cholpon Dzhumakova $^{4}$, Asel Namazbekova $^{5}$

\section{AFFILIATIONS}

${ }^{1}$ Department of Pathology, International Higher School of Medicine, International University of Kyrgyzstan, Bishkek, Kyrgyzstan

${ }^{2}$ Department of Public Health and Healthcare, I.K. Akhunbaev Kyrgyz State Medical Academy, Bishkek, Kyrgyzstan

${ }^{3}$ Department of Experimental Pathophysiology, Kyrgyz-Russian Slavic University, Bishkek, Kyrgyzstan

${ }^{4}$ Department of Gastroenterology, National Center of Oncology and Hematology, Bishkek, Kyrgyzstan

${ }^{5}$ Department of Cancer registry, National Center of Oncology and Hematology, Bishkek, Kyrgyzstan

*Corresponding author

Name: Vityala Yethindra

Email:yethindravityala10@gmail.com

Contact: +919121925658

Key-words: Neurology, Genetics, Ophthalmology, Paediatrics and adolescent medicine. 


\section{KEY CLINICAL MESSAGE}

This paper details a case of neurofibromatosis type 1 (NF1) in a genotype-phenotype correlation, the complexity of pathogenic variants of NF1 gene make correlation difficult. Establishing correlation is useful for targeted therapeutic intervention.

\section{INTRODUCTION}

Neurofibromatosis type 1 (NF1), a common autosomal dominant Mendelian disorder, has an unpredictable presentation with a wide spectrum of inter- and intra-family clinical variability. ${ }^{1}$ With an incidence of one in $3000,{ }^{2}$ it is caused by loss-of-function pathogenic variants of the NF1 tumor suppressor gene, which is located on chromosome $17 \mathrm{q} 11.2$ and encodes neurofibromin, a negative regulator of RAS proteins. ${ }^{3}$

For its diagnosis, at least two of the following criteria must be satisfied: presence of $>6$ café-au-lait spots or hyperpigmented macules $(>5 \mathrm{~mm}$ and $>15 \mathrm{~mm}$ in diameter in prepubertal and postpubertal children, respectively); $>2$ neurofibromas or one plexiform neurofibroma (pNF); $>2$ inguinal and axillary freckles; optic nerve glioma; two or more Lisch nodules; sphenoid dysplasia or typical long bone abnormalities such as pseudarthrosis; and a first-degree relative with NF1. ${ }^{3}$ Children with a severe phenotype have cutaneous neurofibromas (cNF) or pNF, gliomas of the optic pathway, and peripheral nerve sheath tumors. ${ }^{4}$

The complexity and diversity of pathogenic variants of NF1 gene make genotype-phenotype correlations very difficult. Establishing this association is potentially useful for targeted therapeutic intervention.

\section{CASE PRESENTATION}

A six-year-old boy, an only child of a healthy and non-consanguineous couple, presented with dysmorphic facial features - arched eyebrows, downward slanting eyes, myopathic face, hypertelorism, broad nasal root, strabismus, low set ears and micrognathia, multiple café-au-lait spots (> 50), a cNF, and delayed psychomotor development from the neonatal period.

During follow-up, there was an increase in the number of cNF $(>20)$ and development of numerous neurofibromas in the nerve pathways, including the intercostal and pelvic plexus. He also presented with a pNF that affected the upper limb when he was seven years old. At four years of age, he had been diagnosed with a Lisch nodule in his right eye. The General Intelligence Assessment results for cognitive ability was 66\%, validating the diagnosis of mental retardation.

Brain magnetic resonance imaging demonstrated small hamartomas in the globus pallidus, thalamus, periaqueductal gray, splenium of the corpus callosum (right), deep ipsilateral cerebellar hemisphere, and pons, no mass effect. Abdominal computed tomography revealed a $89 \mathrm{~mm} \times 56 \mathrm{~mm}$ retroperitoneal mass, affecting the great visceral vessels, but with no occlusion, and exerting a mass effect on the adjacent structures. For the etiological diagnosis, sequencing and Multiplex Ligation-dependent Probe Amplification analysis of the NF1 gene was carried out that revealed a heterozygous deletion of the complete coding sequence of the gene.

Chromosomal microarray test revealed a submicroscopic deletion on the long arm of chromosome 17 (band 17q11.2). This was an uncommon type 2 deletion of $1.2 \mathrm{Mb}$ that was generated by non-allelic homologous recombination, with breakpoints located within the SUZ12 gene and its SUZ12P pseudogene.

\section{DISCUSSION}

NF1 has a variable presentation that is dependent on age, making it difficult to establish genotype-phenotype correlations. ${ }^{5}$ Although there have been significant developments in the research on NF1 underlying this phenotypic heterogeneity are unknown. Only a small number of genetic abnormalities have been correlated with a characteristic phenotype. ${ }^{6,7}$

A large number of different mutations have been reported, but submicroscopic deletions, splicing mutations, and stop mutations are the most common. Submicroscopic deletions are responsible for $5-10 \%$ of reported cases of NF1 and phenotypic variability is seen in these patients because of differences in the size of the deletion. ${ }^{6,7}$ Chromosome 17 submicroscopic deletions are associated with a more severe phenotype, as seen 
in this patient. This phenotype includes a high number of $\mathrm{cNF}$ and $\mathrm{pNF}$ at neonatal age, dysmorphic facial features, intellectual disability, Lisch nodules, and multiple brain hamartomas. However, our patient did not present with other characteristic symptoms, such as somatic overgrowth, skeletal abnormalities, connective tissue disorders, and cardiovascular malformations, which are associated with submicroscopic deletions.

Although many different mutations associated with neurofibromatosis have been reported, information on genotype-phenotype correlations remains limited. ${ }^{8,9}$ The extreme variability in the clinical presentation of NF1 makes these correlations difficult, although they are crucial for the patient and his family.

The presence of a more severe phenotype warrants the search for submicroscopic deletions and a precise monitoring of complications.

\section{AUTHOR CONTRIBUTIONS}

Vityala Yethindra - Involved in conception, design of the work, manuscript preparation, and data acquisition.

Tugolbai Tagaev - Involved in clinical management.

Elmira Mainazarova - Involved in manuscript review and clinical management

Cholpon Dzhumakova - Involved in manuscript preparation and data acquisition.

Asel Namazbekova - Involved in conception of the work and manuscript review.

\section{ACKNOWLEDGMENTS}

The authors acknowledge their patient for kind cooperation and for providing consent.

\section{CONFLICT OF INTEREST}

The authors declare no conflicts of interest.

\section{ETHICS APPROVAL AND CONSENT TO PARTICIPATE}

Signed consent was obtained from the patient for this case report. The patient was also made aware that the information taken were used for publication.

\section{ABSTRACT}

We are reporting a case of neurofibromatosis type 1 in a genotype-phenotype correlation and chromosomal microarray test revealed a submicroscopic deletion on the long arm of chromosome 17, which is associated with a more severe phenotype. The presence of a more severe phenotype warrants precise monitoring of complications.

\section{REFERENCES}

1. Williams VC, Lucas J, Babcock MA, Gutmann DH, Korf B, Maria BL. Neurofibromatosis type 1 revisited. Pediatrics. 2009; 123: 124-33.

2. Evans DG, Howard E, Giblin C, Clancy T, Spencer H, Huson SM, et al. Birth incidence and prevalence of tumor-prone syndromes: estimates from a UK family genetic register service. Am J Med Genet A.2010; 152A: 327-32.

3. Koczkowska M, Chen Y, Callens T, Gomes A, Sharp A, Johnson S, et al. Genotype-phenotype correlation in NF1: evidence for a more severe phenotype associated with missense mutations affecting NF1 codons 844-848. Am J Hum Genet . 2018; 102: 69-87.

4. Batalla A, Iglesias-Puzas A, Freire-Bruno J, Herrero-Hermida J, Flórez A. Genotype-phenotype correlation in type 1 neurofibromatosis: pMet992del mutation and milder disease. Pediatr Dermatol. 2018; 35: e268-71.

5. Van Minkelen R, Van Bever Y, Kromosoeto JN, Withagen-Hermans CJ, Nieuwlaat A, Halley DJ, et al. A clinical and genetic overview of 18 years neuro-fibromatosis type 1 molecular diagnostics in the Netherlands. Clin Genet. 2014; 85: 318-27. 
6. Kehrer-Sawatzki H, Mautner VF, Cooper DN. Emerging genotype-phenotype relationships in patients with large NF1 deletions. Hum Genet.2017; 136: 349-76.

7. Barrea C, Vaessen S, Bulk S, Harvengt J, Misson JP. Phenotype-genotype correlation in children with neurofibromatosis type 1. Neuropediatrics. 2018; 49: 180-4.

8. Mao B, Chen S, Chen X, Yu X, Zhai X, Yang T, et al. Clinical characteristics and spectrum of NF1 mutations in 12 unrelated Chinese families with neurofibromatosis type 1. BMC Med Genet. 2018; 19: 101.

9. Pasmant E, Vidaud M, Vidaud D, Wolkenstein P. Neurofibromatosis type 1: from genotype to phenotype. J Med Genet. 2012; 49: 483-9. 